\title{
Collagenase digestion of bone marrow trephine biopsy specimens: an important adjunct to haematological diagnosis when marrow aspiration fails
}

\author{
Z T Maung, N P Bown, P J Hamilton
}

\begin{abstract}
Failure to obtain sufficient material from marrow aspiration (dry tap) posed a diagnostic problem in two patients with pancytopenia. By using collagenase digestion of the trephine biopsy specimen, a precise diagnosis was reached. This technique is very useful because it permits flow cytometric and immunocytochemical analyses of cell suspensions obtained after collagenase digestion of the trephine biopsy specimen core. Acute leukaemia presenting with a dry tap can therefore be accurately immunophenotyped. The technique is easy to perform and merits wider use.
\end{abstract}

(F Clin Pathol 1993;46:576-577)

Conventional cytomorphological analysis of Romanowsky stained bone marrow smears is frequently complemented by cytochemical, immunophenotypic, and cytogenetic studies for complete diagnostic, therapeutic, and prognostic information. But sometimes bone marrow aspiration fails and a dry tap results. Collagenase digestion of a trephine biopsy specimen is easily performed in such situations and results in the garnering of enough cells in a suspension to permit meaningful cytomorphology and immunophenotyping and rapid arrival at a precise diagnosis. ${ }^{1}$

\section{Methods}

A modification of the original method of Mononen and Jansson was used. ${ }^{1}$ A $2 \mathrm{~mm}$ long piece of bone marrow trephine obtained using a Jamshidi needle was cut off the parent biopsy specimen core and incubated for 1 hour at $37^{\circ} \mathrm{C}$ with 2 units of collagenase (Sigma C2799) in $400 \mu \mathrm{l}$ of RPMI 1640 medium (Northumbria Biologicals) containing L-glutamine $2 \mathrm{mM}$, fetal calf serum $10 \%$ (v/v), adjusted to $\mathrm{pH} 7 \cdot 4$ by $1 \mathrm{M} \mathrm{HCl}$. The liberated cells were resuspended in phosphate buffered saline. The cell suspension is suitable for flow cytometry and, after cytocentrifugation, for a range of cytochemical investigations.

\section{Case reports}

CASE 1

A 51 year old man presented with epistaxis and widespread purpura. A blood count showed that the haemoglobin was $59 \mathrm{~g} / 1$, mean cell volume $101 \mathrm{fl}$, white cell count $2.9 \times 10^{9} / 1$, and platelets $8 \times 10^{9} / 1$. There were no blasts in the peripheral blood smear. Plasma $B_{12}$, folate, and red cell folate concentrations were high normal. Dry taps were obtained from both iliac crests. A drop of marrow was expelled from the needle with the stylet and used for cytogenetic analysis. A trephine biopsy specimen was obtained and imprints of the core showed only bare nuclei with no morphological detail. The core was bisected, one part sent for histological examination and the other for collagenase digestion. The digest was cytocentrifuged and stained with May-Grünwald-Giemsa (MGG). This showed multinuclearity and delayed nuclear condensation of erythroid precursors. Myelopoiesis was morphologically unremarkable with no excess of blasts. Histological examination of the trephine biopsy specimen showed hypercellular marrow with expansion of red and white cell precursors; no megakaryocytes were identified. Reticulin fibres were greatly increased. In the context of severe peripheral pancytopenia and a hypercellular marrow with no evidence of infiltration, the dysplastic, albeit non-specific, appearance of the erythroid cells suggested a primary myelodysplastic syndrome (MDS) associated with increased marrow fibrosis. ${ }^{2}$ The diagnosis of MDS was supported by the cytogenetic findings which included trisomy $6,5 q-$ and $7 p-$ and $21 p+$, well established markers of MDS and myeloid leukaemia. Over the next four months the refractory anaemia evolved with up to $10 \%$ myeloblasts appearing in the blood, the spleen enlarged, and he died from complications of bone marrow failure.

\section{CASE 2}

A 28 year old man presented with purpura. A full blood count showed that the haemoglobin has $45 \mathrm{~g} / \mathrm{l}$, white cell count $6.4 \times 10^{9} / 1$ with blasts $0.1 \times 10^{9} / 1$, and platelets $10 \times$ $10^{\circ} / 1$. Bone marrow aspirate was difficult, yielding a minute quantity of marrow barely efficient for one smear for MGG staining and a few drops for cytogenetic studies. The aspirate smear showed a heavy monotonous infiltration of undifferentiated blasts. Histological examination of the trephine biopsy specimen confirmed acute leukaemia but could not distinguish lymphoblastic from myeloid form. An aliquot of cells liberated by collagenase digestion of the core was analysed by flow cytometry (FACScan, Becton-Dickinson) using a panel of monoclonal antibodies. The 
suspension was also cytocentrifuged on to a glass slide for alkaline phosphatase antialkaline phosphatase (APAAP) immunoenzymatic detection of terminal deoxynucleotidyl transferase (TdT) and cytoplasmic $\mu$ chains. The marker results showed the following TdT 50\%, CD10<1\%, CD19 85\%, CD3 $8 \%, \mathrm{CD} 73 \%, \mathrm{CD} 13$ 7\%, CD33 5\% and cyt- $\mu 80 \%$ positivity. Pre-B cell acute lymphoblastic leukaemia (ALL) was diagnosed. The cytogenetic studies showed an abnormal cell line, 46,XY,der(4)t(1;4)(q2?;q3?). This unbalanced rearrangement resulted in partial trisomy 1q, a common acquired abnormality in a range of malignancies including leukaemias.

\section{Discussion}

About $4 \%$ of bone marrow examinations result in a dry tap, ${ }^{3}$ usually caused by increased fibrosis or dense infiltration by malignant cells. A trephine biopsy specimen is of crucial importance in establishing the true aetiology. There is increasing interest in the diagnosis of bone marrow disorders by histological examination of trephine biopsy specimens, ${ }^{4}$ but errors do occur and we believe there is no substitute for careful examination of smears and suspensions of individual cells. Indeed, a patient with megaloblastic anaemia narrowly escaped a course of chemotherapy prescribed on the basis of biopsy specimen appearances. ${ }^{56}$ It is also difficult to distinguish, morphologically, myeloid from lymphoid malignancy in bone marrow biopsy cores. Examination of trephine imprints may often be helpful, although one study reported that only $27 \%$ of dry taps resulted in material which was diagnostically satisfactory. ${ }^{1}$ This same study first described a superior method for the cytological examination of bone marrow biopsy specimens, applying collagenase digestion and cytocentrifugation. The method is simple, rapid, and within the scope of most haematology laboratories, yet it is underused, often ignored, and must sometimes be rediscovered. ${ }^{3}$

The two cases described here illustrate and re-emphasise the value of this technique. It is always difficult to distinguish fibrotic MDS from idiopathic myelofibrosis by inspection of decalcified, paraffin wax embedded biopsy specimens. Characteristic features of MDS, such as dysmegakaryopoiesis and abnormal localisation of immature precursors (ALIP) ${ }^{7}$ have been described, but they are neither restricted to various FAB subtypes of $\mathrm{MDS}^{8}$ nor easily detected other than in plastic embedded biopsy specimens. The demonstration of dysplastic erythropoiesis by cytology allowed a diagnosis of MDS to be made in case 1 . The clonal nature of the disorder was confirmed by the cytogenetic findings and the haematological progression vindicated the original diagnosis. It has been reported that cells treated with collagenase retain their cytochemical reactivity, allowing a rapid definitive diagnosis of leukaemia. ${ }^{9}$ In case 2 successful use of flow cytometry and immunocytochemistry on collagenase-treated cells made it possible to make a firm diagnosis of pre-B ALL.

Cytogenetic analysis is important for diagnosis, classification, and prognosis of most clonal haematological disorders. Successful cytogenetic analysis of cells from bone marrow biopsy cores has been reported, ${ }^{10}$ but the yield of dividing cells suitable for chromosomal analysis has not been high in our hands. In both cases reported here we obtained cytogenetic information from culturing what little marrow was present in our aspirate needle, relying on obtaining sufficient cells from the collagenase digest for morphological and immunophenotypic studies. The knowledge that useful morphological and immunophenotypic information can be obtained from the digested cores provides the opportunity for more flexible use of the small amount of marrow sometimes obtained in difficult aspirates. There is now little justification for accepting failure to establish a firm diagnosis of leukaemia on the grounds that a difficult aspirate yielded insufficient cells.

We are grateful to the Tyneside Leukaemia Research Association for the flow cytometry facilities.

1 Mononen I, Jansson SE. A rapid procedure, by collagenase treatment and cytocentrifugation, for the cytological evaluation of bone marrow biopsy specimens. Clin Lab Haematol 1986;8:149-54.

2 Lambertenghi-Deliliers G, Orazi A, Luksch R, Annaloro C, Soligo D. Myelodysplastic syndrome with increased marrow fibrosis; A distinct clinico-pathological entity. Br F Haematol 1991;78:161-6.

3 Humphries JE. Dry tap bone marrow aspiration: Clinical significance. Am $\mathcal{F}$ Hematol 1990;35:247-50.

4 Winfield DA, Polacarz SV. Bone marrow histology 3: Value of bone marrow core biopsy in acute leukaemia, myelodysplastic syndromes, and chronic myeloid leukaemia. F Clin Pathol 1992;45:855-9.

5 Dokal IS, Cox TM, Galton DAG. Vitamin $B_{12}$ and folate deficiency presenting as leukaemia. $B r \operatorname{Med~} \mathcal{F} 1990$; 300:1263-4.

6 Lennard AL. Vitamin $B_{12}$ and folate deficiency presenting as leukaemia. Br Med f 1990;301:180.

7 Tricot G, De Wolfe-Peeters C, Hendrickx B, Verwilghen $\mathrm{RL}$. Bone marrow histology in myelodysplastic syndromes I-Histological findings in myelodysplastic syndromes and comparison with bone marrow smears. $\operatorname{Br} \mathcal{F}$ Haematol 1984;57:423-30.

8 Bennett JM, Catovsky D, Daniel MT, et al. The FrenchAmerican-British (FAB) Co-operative group. Proposals for the classification of the myelodysplastic syndromes. Br f Haematol 1982;51:185-99.

9 Gibson J, Grimmett K, Joshua D, Kronenberg H. Collagenase digestion of trephine biopsies: Rapid diagnosis of the dry tap with preservation of cytochemical reactivity. Pathology 1988;20:200-1.

10 Martin P, Rowley JD, Baron J. The use of bone core biopsies for cytogenetic analysis. Hum Genet 1979;51:163-9. 